\title{
How surface magnetism affects helioseismic waves
}

\author{
Paul S. Cally \\ Centre for Stellar and Planetary Astrophysics, Monash University, Clayton, Victoria, Australia \\ 3800 \\ email: paul.cally@sci.monash.edu.au
}

\begin{abstract}
It has been known for two decades that sunspots both absorb and advance the phase of solar $f$ and $p$-modes. More recently, Time-Distance and other local helioseismic techniques have been used to probe active regions by exploring phase shifts which are interpreted as traveltime perturbations. Although absorption is an intrinsically magnetic effect, phase shifts may be produced by both thermal and magnetic effects (and of course flows, though these can be factored out by averaging travel times in opposite directions). We will show how these two effects alter wave phase, and conclude that phase shifts in umbrae are predominantly thermal, whilst those in highly inclined field characteristic of penumbrae are essentially magnetic. The two effects are generally not additive.
\end{abstract}

Keywords. Sun: helioseimology, Sun: magnetic fields

\section{Introduction}

The aim of this paper is to present a simplified view of how strong surface magnetic fields in solar active regions are expected to influence helioseismic waves incident from below, with implications for both the atmosphere above, and the internal helioseismic wave field. As first suggested by the author nearly a decade ago (Cally (2000)), magnetic field inclination is the crucial ingredient that redresses the inability of vertical magnetic field models Cally, Bogdan \& Zweibel (1994) to sufficiently interact with $p$-modes to explain observed absorption and phase shifts. Our understanding of the process of mode transmission/conversion has advanced greatly in the last few years due to the development of several different techniques, most notably Generalized Ray Theory see Section 2. However, analysis of the phase discontinuities characteristic of reflection at caustics and of mode conversion has only been tackled this year (Cally (2009a), Cally (2009b)), and we summarize the results in Section 3.

\section{Transmission and Conversion}

Classical ray theory as applied to MHD waves Weinberg (1962) is based on the eikonal approximation and the resulting dispersion relation $\mathcal{D}\left(\omega, k_{x}, k_{y}, k_{z} ; \mathbf{x}\right)=0$ connecting the frequency $\omega$, wave vector $\mathbf{k}$, and position $\mathbf{x}$. With $\omega, k_{x}$ and $k_{y}$ held fixed for instance (in a horizontally invariant atmosphere), $k_{z}$ changes with height $z$ in such a way that $\mathcal{D}\left(\omega, k_{x}, k_{y}, k_{z} ; z\right)=0$, thus defining a relationship between $k_{z}$ and $z$. This forms loci in the $z-k_{z}$ plane which may be categorized as fast, slow, or Alfvén, and generally these are disjoint, meaning that solutions stay fast, slow, or Alfvén. However, at avoided crossings between these curves (mode transmission/conversion points), the eikonal approximation breaks down and a more sophisticated matching process Tracy, Kaufman \& Brizard (2003) is required which reveals tunnelling between the branches. Fast/slow transmission occurs most readily when the attack angle between the wave vector $\mathbf{k}$ and the magnetic field $\mathbf{B}$ at the Alfvén/acoustic equipartition level $a=c$ is small; this represents the coupling of a predominantly acoustic wave below $a=c$ with a predominantly acoustic wave above this level, hence "transmission". At large attack angle a fast wave from below stays fast as it passes through $a=c$, but that corresponds to a change of nature from 
acoustic to magnetic, hence "conversion". At intermediate attack angles there is both substantial transmission and conversion: the wave splits.

If we are concerned with the part of the split wave field which returns to the solar interior as part of the "helioseismic field" of skipping acoustic waves, its path through the magnetic surface layer predominantly follows this route: (i) conversion to a magnetic (i.e., fast) wave near $a=c$; (ii) refraction back downward due to the rapidly increasing Alfvén speed with height in the active region atmosphere; (iii) conversion back to acoustic as it passes downward through $a=c$ again. All this is most pronounced if the attack angle is large. Since helioseismic waves typically impinge on the surface quite steeply, this process is most favoured in highly inclined magnetic field, characteristic of sunspot penumbrae. In umbrae, where the field is more vertical and the attack angle smaller, there is mostly transmission as an acoustically dominated atmospheric wave, which may or may not reflect, depending on the relation of the wave frequency to the acoustic cutoff frequency. Even if it does though, the wave timings, both group and phase, are very different for the two scenarios.

\section{Conclusions: Phase and travel-time perturbation}

Local helioseismology typically identifies phase differences between skips in active regions compared with equivalent oscillations in the quiet Sun as evidence of a travel time perturbation. These may be variously identified as resulting from changes in subsurface sound speed, flows, or direct magnetic effects. Numerical modelling, both wave and ray, leads to the following conclusions regarding ray timings based on phase perturbations:

(a) There is a strong field inclination dichotomy;

(b) Thermal effects are only a good predictor of travel-time perturbations (really phase perturbations) in umbrae, where field is near vertical and waves are impinging roughly parallel to $\mathbf{B}$;

(c) Travel-time perturbations in highly inclined field (penumbra) are mostly magnetic in origin;

(d) Magnetic effects increase strongly with $|B|$.

This travel time dichotomy may explain the observations of Couvidat \& Rajaguru (2007), who notice a distinct "ring-like" structure in travel time maps in sunspot penumbrae.

\section{References}

Cally, P. S. 2000, Solar Phys., 192, 395

Cally, P. S. 2006, Phil. Trans. Roy. Soc. Lond. A 364, 333

Cally, P. S. 2007, $A N$ 328, 286

Cally, P. S. 2009a, Solar Phys. 254, 241

Cally, P. S. 2009b, MNRAS 395, 1309

Cally, P. S., Bogdan, T. J., \& Zweibel, E. G. 1994, ApJ 437, 505

Couvidat, S. \& Rajaguru, S. P. 2007, Astrophys. J. 661, 558

Hansen, S. C. \& Cally, P. S. 2009, SolarPhys. 255, 193

Schunker, H. \& Cally, P. S. 2006, MNRAS, 372, 551

Tracy, E. R., Kaufman, A. N., \& Brizard, A. J. 2003, Phys. Plasmas 10, 2147

Weinberg, S. 1962, Phys. Rev., 126, 1899 